\title{
Cerebral Hypometabolism in Progressive Supranuclear Palsy Studied with Positron Emission Tomography
}

\author{
Norman L. Foster, MD, ${ }^{*}$ Sid Gilman, MD, ${ }^{*}$ Stanley Berent, $\mathrm{PhD},{ }^{* \dagger} \dagger$ Elizabeth M. Morin, BS,* \\ Morton B. Brown, $\mathrm{PhD}, \ddagger$ and Robert A. Koeppe, $\mathrm{PhD} \S$
}

\begin{abstract}
Progressive supranuclear palsy (PSP) is characterized by supranuclear palsy of gaze, axial dystonia, bradykinesia, rigidity, and a progressive dementia. Pathological changes in this disorder are generally restricted to subcortical structures, yet the type and range of cognitive deficits suggest the involvement of many cerebral regions. We examined the extent of functional impairment to cerebral cortical and subcortical structures as measured by the level of glucose metabolic activity at rest. Fourteen patients with PSP were compared to 21 normal volunteers of similar age using ${ }^{18} \mathrm{~F}$ 2-fluoro-2-deoxy-D-glucose and positron emission tomography. Glucose metabolism was reduced in the caudate nucleus, putamen, thalamus, pons, and cerebral cortex, but not in the cerebellum in the patients with PSP as compared to the normal subjects. Analysis of individual brain regions revealed significant declines in cerebral glucose utilization in most regions throughout the cerebral cortex, particularly those in the superior half of the frontal lobe. Declines in the most affected regions of cerebral cortex were greater than those in any single subcortical structure. Although using conventional neuropathological techniques the cerebral cortex appears to be unaffected in PSP, significant and pervasive functional impairments in both cortical and subcortical structures are present. These observations help to account for the consrellation of cognitive symptoms in individual patients with PSP and the difficulty encountered in identifying a characteristic psychometric profile for this group of patients.
\end{abstract}

Foster NL, Gilman S, Berent S, Morin EM, Brown MB, Koeppe RA. Cerebral hypometabolism in progressive supranuclear palsy studied with positron emission tomography. Ann Neurol 1988;24:399-406

Progressive supranuclear palsy (PSP) is characterized by supranuclear palsy of gaze, axial dystonia, pseudobulbar palsy with dysarthria and dysphagia, bradykinesia and rigidity, and a progressive dementia [1-5]. Typical pathological features of neuronal loss, distinctive neurofibrillary tangles, and gliosis most conspicuously affect the globus pallidus, subthalamic nucleus, red nucleus, substantia nigra, dentate nucleus, tectum, and periaqueductal gray matter and are limited to subcortical structures; the cerebral cortex generally appears to be unaffected with conventional neuropathological techniques $[1,6]$. Despite the apparent lack of cerebral cortical involvement, behavioral changes and dementia are frequent concomitants of this disorder $[1,7,8]$. This implies that there may be impairment of cerebral cortical function. Previous studies with ${ }^{18} \mathrm{~F}$-2-fluoro-2-deoxy-D-glucose $\left({ }^{18} \mathrm{~F}\right.$-FDG $)$ and positron emission tomography (PET) have demonstrated glucose hypometabolism most prominently in the frontal cortex [9-11]. A disturbance in function of the frontal lobes only, however, fails to explain the occurrence in patients with PSP of other symptoms of intellectual decline such as memory impairment, anomia, and visuospatial disturbance, which have classically been localized to other cortical regions. We therefore examined glucose metabolism throughout the cerebral cortex. We also studied metabolic activity in the cerebellum and in subcortical structures that have prominent neuronal loss in this disease, such as the caudate nucleus, putamen, thalamus, and brainstem.

\section{Methods \\ Patient Selection}

Fourteen patients with typical features of PSP ( 7 men, 7 women, mean age $66 \pm 6$, range $59-75$ years) and 21 normal control subjects ( 13 men, 8 women, mean age $60 \pm 8$, range $46-71$ years) were studied. All had cranial computed tomographic (CT) scans showing no localized abnormalities and had not taken any medication with central nervous system effects for at least 2 weeks before the PET study. None
From the Departments of *Neurology, †Psychiatry and Psychology, $\ddagger$ Biostatistics, and the $§$ Division of Nuclear Medicine, The University of Michigan, Ann Arbor, MI.

Received Jan 20, 1988, and in revised form Mar 31. Accepted for publication Apr 3, 1988.
Address correspondence to Dr Foster, Department of Neurology, The University of Michigan, 1920 Taubman, Box 0316, 1500 East Medical Center Dr, Ann Arbor, MI 48109-0316. 


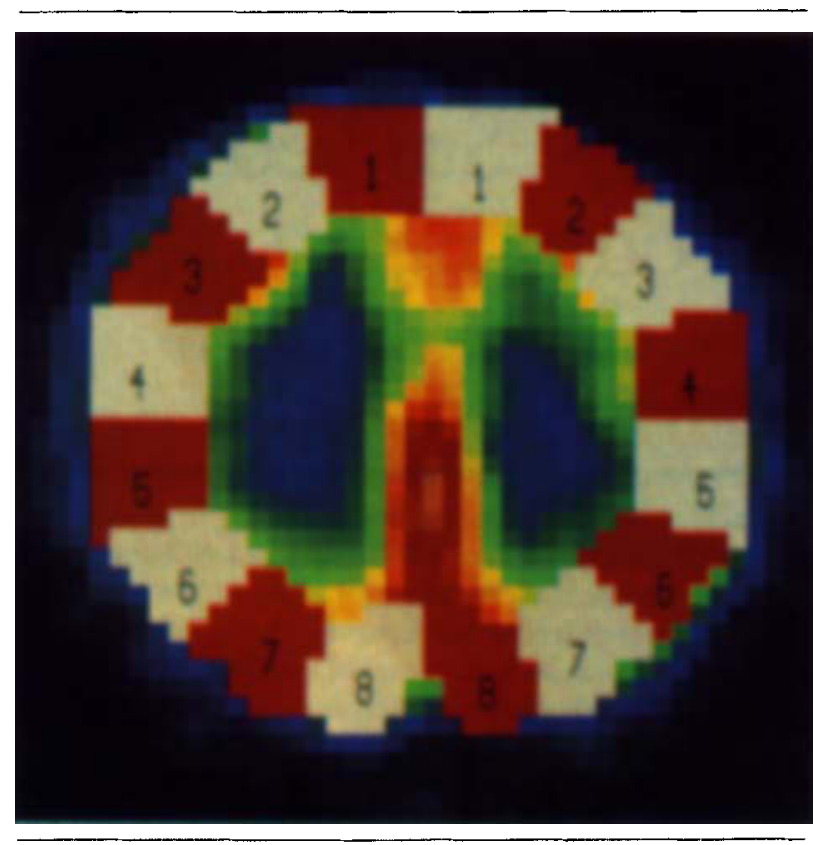

Fig 1. Regions of cerebral cortex as defined by the automated image analysis program in a typical horizontal slice from a normal subject. Mean metabolic rates from bomologous regions of left and right cortex extending from region 1, most anterior, to region numbered 8 , most posterior, were analyzed to determine localized changes in patients with progressive supranuclear palsy (PSP) as compared with control subjects. This image corresponds to the most superior borizontal slice of the brain analyzed, designated as level $F$

had a history of significant head injury or evidence of stroke. The diagnosis of PSP was based on the presence of supranuclear gaze palsy, pseudobulbar palsy, axial and extremity rigidity, and dementia. One patient with this diagnosis has subsequently come to postmortem examination and this revealed multiple system degeneration. Since this patient fulfilled the clinical eligibility criteria for this study his data are included. The normal control subjects were healthy individuals without evidence of neurological or psychiatric illness who had been recruited by advertisement from the community.

\section{PET Method}

These studies were approved by the institutional review board and informed consent was obtained from each subject. Subjects were scanned while at rest with eyes patched and ears open to ambient noise in a quiet room. PET images were obtained with a TCC PCT-4600A three-ring, five-slice tomograph (Cyclotron Corporation, Berkeley, CA) following intravenous administration of 5 to $10 \mathrm{mCi}$ of ${ }^{18} \mathrm{~F}-\mathrm{FDG}$. ${ }^{18}$ F-FDG was synthesized using the reaction of acetyl hypofluorite with triacetylglucal in Freon-11 [12]. This method results in less than $5 \%$ contamination with 2-deoxy-2-fluoromannose. In each scanning session, a series of four interleaved scans was obtained parallel to the canthomeatal line. This results in up to 20 images with an in-plane resolution of $11 \mathrm{~mm}$ full width at half maximum (FWHM) and an axial resolution of $9.5 \mathrm{~mm}$ FWHM. Serial arterial blood sam-

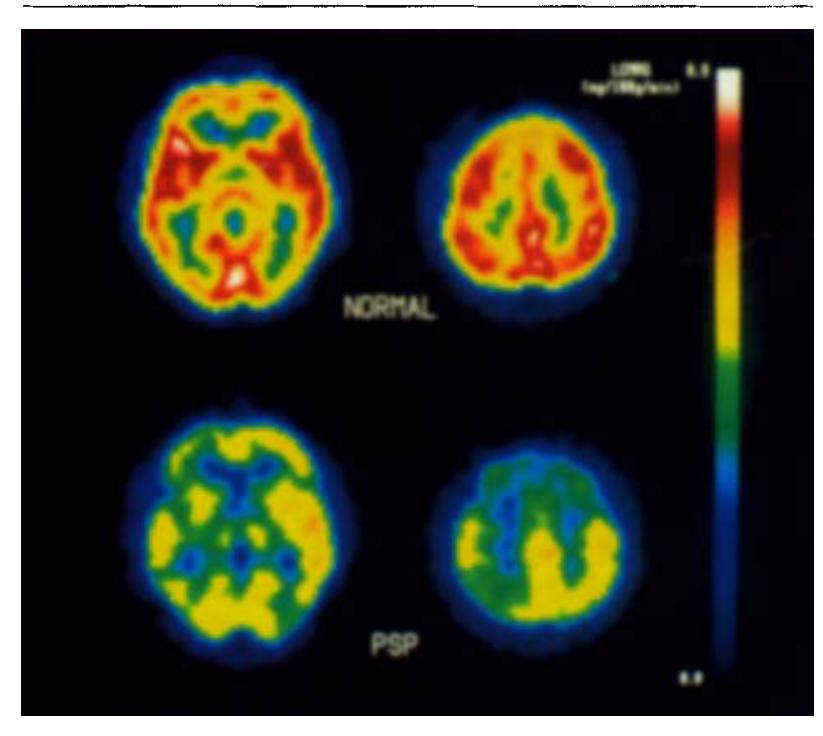

Fig 2. Positron emission tomographic scan images following ${ }^{18} \mathrm{~F}$ 2-fluoro-2-deoxy-D-glucose administration in a typical normal subject (top images) and a typical patient with progressive supranuclear palsy (bottom images). Each is a borizontal section in a plane parallel to and approximately $4 \mathrm{~cm}$, level $A$ (left images) or $8 \mathrm{~cm}$, level $F$ (right images) above the cantbomeatal line. The front of the bead is to the top of the image and the right of the bead is to the right of the image. The color bar is the scale used to indicate the rates of glucose utilization $(\mathrm{mg} / 100$ $\mathrm{gm} / \mathrm{min}$ ) with the highest values at the top and lowest values at the bottom of the bar.

ples were used to determine ${ }^{18} \mathrm{~F}-\mathrm{FDG}$ and glucose concentrations. Images were reconstructed following attenuation correction by the standard ellipse method, and local rates of glucose metabolism were calculated using a three-compartment, four-rate constant model with gray matter kineticrate constants derived from normal individuals [13]

\section{Image Analysis}

Local cerebral metabolic rate for glucose (ICMRGlc) in the cerebral cortex was determined using an automated image analysis program that enhances the contrast in the images, mathematically defines the extent of the gray matter, and then selects a region extending no more than $22 \mathrm{~mm}$ inward from the cortical rim, thereby excluding subcortical gray matter. In each patient, 6 horizontal slices extending from the basal ganglia to the top of the centrum semiovale at visually equivalent levels were analyzed. Rates of glucose metabolism were measured in 16 regions of each slice ( 8 in each hemisphere) representing equal sectors of the cortex (Fig 1). Mean cerebral cortical metabolism was determined by averaging rates in all regions analyzed. Mean values in each hemisphere were similarly determined. Therefore, hemispheric rates reflect only the contribution of the cerebral cortical gray matter and do not include subcortical structures, white matter, or ventricles. For simplicity of presentation, regional analysis was performed with the means of values obtained from homologous regions of the left and right hemispheres. Values in each brain region for patients with 
PSP were expressed as a ratio to the mean in that region for normal subjects.

For the analysis of ICMRGlc in the basal ganglia, the image in which the head of the caudate nucleus showed the highest metabolic rates as determined visually from unsmoothed images was selected for measurement of rates in the caudate nucleus and putamen. This same image was generally also used for measurement of metabolic rate of the thalamus. Occasionally, however, the thalamus showed its highest metabolic rates in a slightly more superior image. When this occurred, the second image was used for analyzing thalamic metabolic rates. For each image selected, rates were measured in $7.5 \times 11 \mathrm{~mm}$ regions centered on (1) the head of the caudate nucleus, approximately one-third of the anterior-posterior extent of the cortex and just posterior to the frontal horns of the lateral ventricles; (2) the putamen, halfway between the caudate cross-section and the anterior extent of the thalamus; and (3) the middle of the thalamus. Positioning of the region was assisted by the use of a histogram showing the rates in successive cross-sections of the image. Mean metabolic rates of the caudate nucleus, putamen, and thalamus consisted of the average of rates determined for the right and left nucleus in each individual.

In the cerebellar hemispheres, vermis, and brainstem ICMRGIc was measured in a region centered over a local peak of activity on the image [14]. A $22 \times 11 \mathrm{~mm}$ parallelogram was used for each cerebellar hemisphere, an $11 \times$ $18 \mathrm{~mm}$ rectangle for the vermis, and an $11 \times 15 \mathrm{~mm}$ rectangle was used for the brainstem. Data were obtained from each image (generally 2 or 3 ) containing the cerebellum and brainstem and represent the means of values in all images. Data were obtained from the cerebellar vermis by centering the region of interest over the peak activity seen posterior to the brainstem in the midline. The brainstem region chiefly reflects the pons, but the mesencephalon or medulla oblongata could be partially represented

All estimates of ICMRGlc are expressed as mean \pm standard deviation (SD). Differences between normal and PSP rates were assessed by a two-sample Behrens-Fisher $t$ test (which does not assume equality of variances). The effects of hemisphere, region, and level within a group were tested by a three-way repeated measures analysis of variance (ANOVA).

\section{Results}

PET images of glucose metabolic rates obtained from patients with PSP differed noticeably in appearance from those obtained in normal subjects (Fig 2). Regional analysis demonstrated that patients with PSP had lower glucose metabolic rates in the cerebral cortex, caudate nucleus, putamen, thalamus, and brainstem (Table 1, Figs 3-5). There was also a tendency for cerebellar hemispheric rates to be lower in those with PSP than in the normal subjects, but rates in the cerebellar vermis were unchanged. Significant declines in ICMRGIc were present throughout the cerebral cortex. Anterior brain regions, particularly those in the superior half of the cortex, were most impaired (Fig 6). ANOVA indicated that there was a significant effect

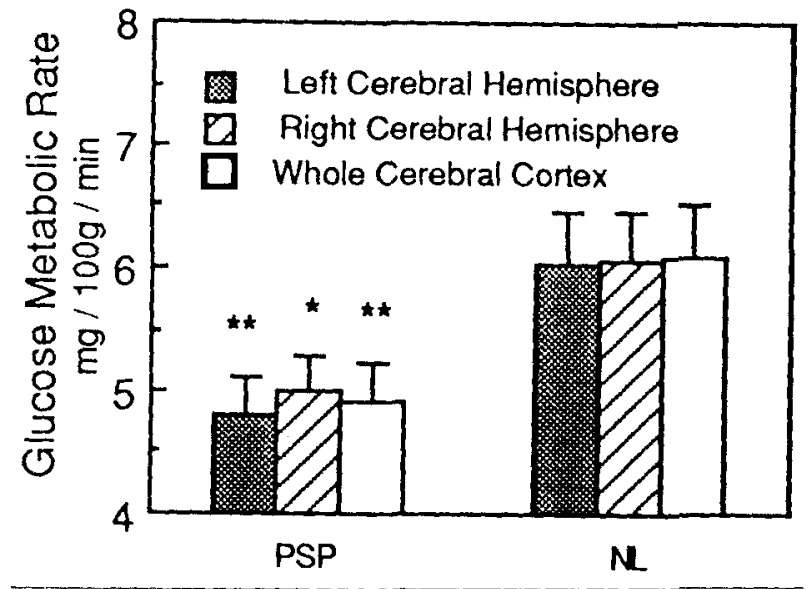

Fig 3. Comparison of mean rates of cerebral cortical glucose metabolism in patients with progressive supranuclear palsy (PSP) and in normal subjects (NL). Hemispheric rates represent the metabolism of the cerebral cortex in each bemisphere. Error bars represent $95 \%$ confidence intervals for the mean. $\left(^{*}=\mathrm{p}=\right.$ $0.0002 ; * *=\mathrm{p}=0.0001$.)

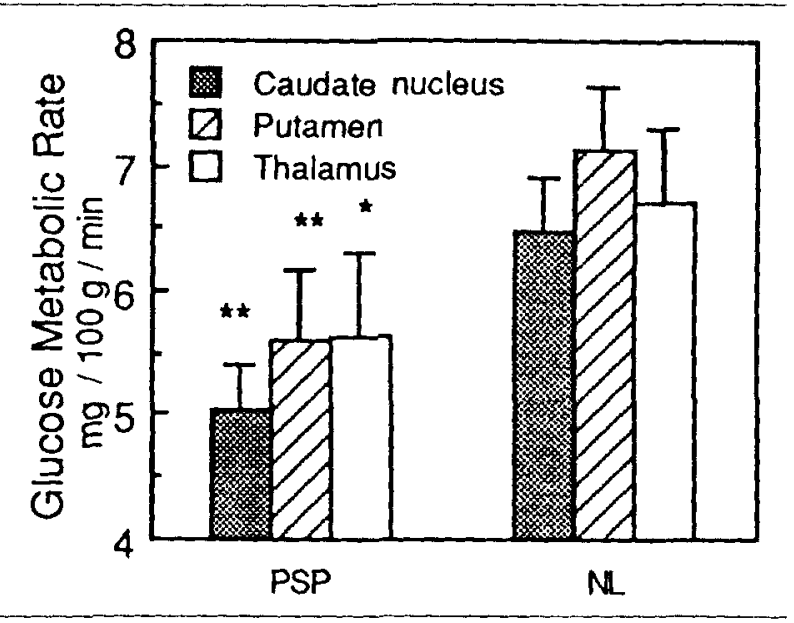

Fig 4. Comparison of mean rates of glucose metabolism in the caudate nucleus, putamen, and thalamus in patients with progressive supranuclear palsy (PSP) and in normal subjects (NL). Error bars represent $95 \%$ confidence intervals for the mean. $\left(^{*}=\right.$ $\mathrm{p}<0.02 ;^{* *}=\mathrm{p}<0.0002$.)

of both level $(p<0.0001)$ and region $(p<0.0001)$ in the patients with PSP. This effect was larger for region than for level. Relative to the rates seen in normal subjects, the declines were greatest in the superioranterior frontal regions, followed by the caudate nucleus, putamen, thalamus, brainstem, and most other areas of the cerebral cortex (see Table 1, Fig 6). Cerebellar hemispheres and vermis were least affected

No significant asymmetries were observed in the ICMRGlc for the caudate nucleus, putamen, or thalamus in either patients or normal subjects; however, the cerebral cortex, which was symmetrical in 


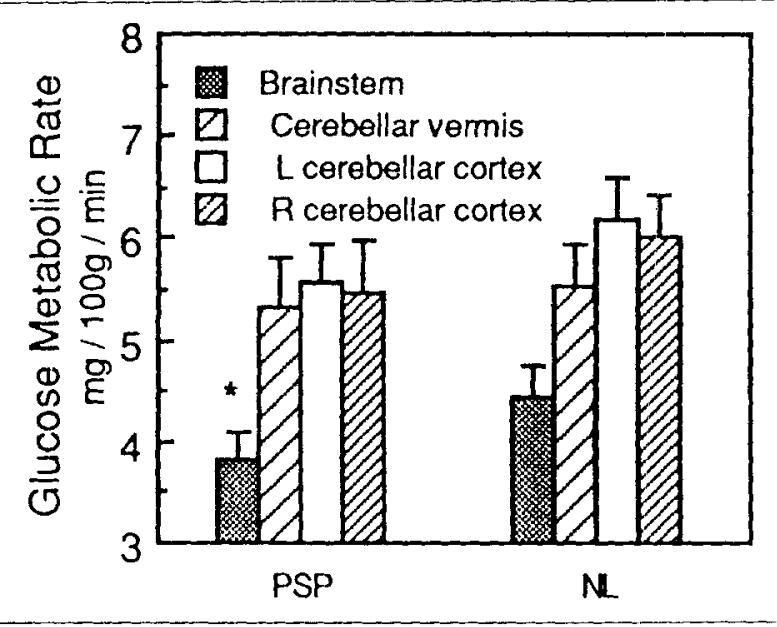

Fig S. Comparison of mean rates of glucose metabolism in brainstem, cerebellar vermis, and bemispheres in patients with progressive supranuclear palsy (PSP) and in normal subjects (NL). Error bars represent $95 \%$ confidence intervals for the mean. ${ }^{*}=\mathrm{p}$ $=0.004$.

normal subjects, was decreased in the left hemisphere (as compared to the right) in patients with PSP. Significant asymmetry of cerebellar hemisphere metabolism was observed in normal subjects, but not in patients with PSP (Table 2).

It was not possible to match our patient group to our cohort of aged normal subjects for both age and sex because the normal subjects are used for many different studies at our center. Consequently, the effects of age and sex were investigated in both the normal subjects and patients. There was no correlation of age with ICMRGlc, and no significant differences in ICMRGIc were observed between the sexes in either the patient or control groups.

\section{Discussion}

The present study demonstrates that neuronal function as measured by regional glucose metabolism is im- paired not only in the frontal region of the cerebral cortex as described previously [9], but also in the remainder of the cerebral cortex as well as in the brainstem, basal ganglia, and thalamus in patients with PSP as compared to normal subjects of similar age. Cerebellar neuronal function shows little change, but the resolution of our PET scanner is not sufficient to discriminate the metabolic activity of the dentate nucleus, where substantial abnormality has been described [1, 3, 15]. Our findings using ${ }^{18} \mathrm{~F}-\mathrm{FDG}$ and PET differ somewhat from the findings anticipated from the pattern of neuronal loss observed at postmortem, but remain consistent with the known neuroanatomy of regions that are most affected in PSP. The ICMRGlc reflects functional activity of neurons, part of which is attributable to the metabolic demands of maintaining cellular integrity and part to those of synaptic activity [16]. Thus, decrements in ICMRGlc can be due to loss of neurons, loss of afferent fibers, or a combination of both. Different brain regions may reflect these mechanisms in different proportions. Abnormalities of glucose metabolism at sites distant from the ultimate structural lesion have been recognized in a variety of circumstances [17], and this phenomenon may account for some of the declines in ICMRGlc seen in patients with PSP.

By light microscopy, the loss of neurons in PSP is most severe in the midbrain tectum, including the superior colliculi, substantia nigra, basal pontine nuclei, and periaqueductal gray matter. Less severe neuronal loss is also seen in the red nucleus and locus ceruleus $[1,3,15]$. In some patients, motor cranial nuclei $[18,19]$ are also affected. The resolution of the PET scanner used in our studies is insufficient to discriminate these structures individually; however, neuronal loss is still the most likely explanation for the decline of ICMRGlc observed in the brainstem.

The globus pallidus, subthalamic nucleus, and medial periventricular portions of the thalamus show

Table 1. Regional Glucose Metabolic Rates $(\mathrm{mg} / 100 \mathrm{gm} / \mathrm{min})$ in Patients with Progressite Supranudear Palsy and Normal Control Subjectsis

\begin{tabular}{lllll}
\hline Brain Region & $\begin{array}{l}\text { Normal Control } \\
\text { Subjects } \\
(\mathrm{n}=21)\end{array}$ & $\begin{array}{l}\text { PSP } \\
(\mathrm{n}=14)\end{array}$ & PSP/Normal & $p$ Value \\
\hline Cerebral cortex & $6.08 \pm 0.94$ & $4.92 \pm 0.52$ & 0.81 & 0.0001 \\
Cerebellar cortex & $6.10 \pm 0.90$ & $5.52 \pm 0.74$ & 0.91 & 0.048 \\
Cerebellar vermis & $5.54 \pm 0.87$ & $5.33 \pm 0.84$ & 0.96 & 0.49 \\
Brainstem & $4.46 \pm 0.67$ & $3.81 \pm 0.49$ & 0.86 & 0.0024 \\
Caudate & $6.48 \pm 0.91$ & $5.04 \pm 0.63$ & 0.78 & 0.00005 \\
Putamen & $7.14 \pm 1.09$ & $5.60 \pm 0.99$ & 0.79 & 0.0002 \\
Thalamus & $6.69 \pm 1.29$ & $5.65 \pm 1.15$ & 0.84 & 0.018 \\
\hline
\end{tabular}

${ }^{a}$ Values represent means \pm standard deviations; $p$ values are for a test of equality of means by the Behrens-Fisher 1 test, which is equivalent to a test that the PSP/normal ratio is unity.

PSP $=$ progressive supranuclear palsy. 


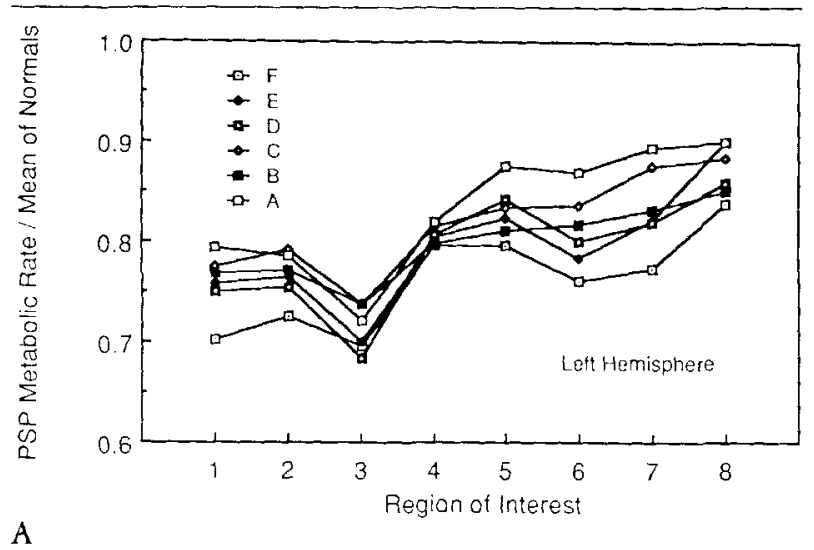

A

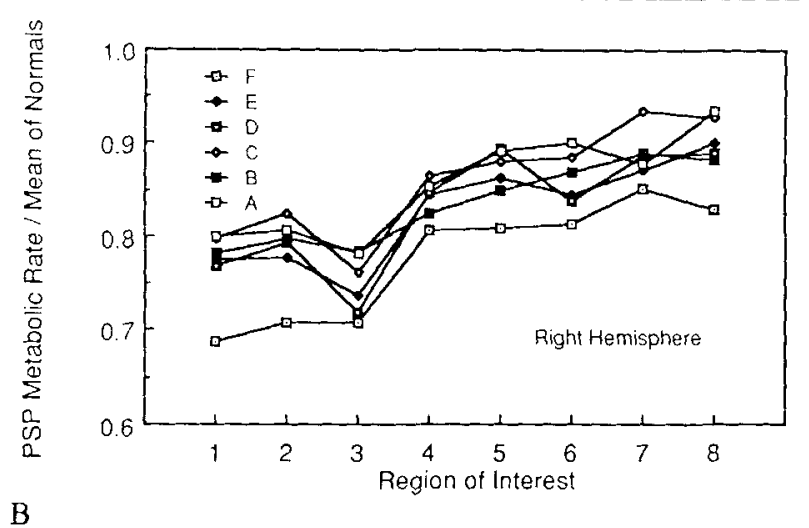

Fig 6. Regional cerebral cortical metabolic rates for glucose in the left hemisphere $(A)$ and in the right bemisphere $(B)$ in patients with progressive supranuclear palsy (PSP) expressed as a ratio to the mean rate observed in the same region in normals. Horizontal sections compared are labeled from $A$, most inferior, to $F$, most superior. Regions of interest extend from the most anterior (1) to the most posterior (8). considerable neuronal loss in PSP, although generally more neuronal loss is found in the brainstem $[1,3]$. Other portions of the thalamus, caudate nucleus, and putamen are less affected. Neurons in the striatum that contain postsynaptic dopamine receptors are damaged $[20,21]$, and important afferents are lost. Dopaminergic fibers from the substantia nigra degenerate, leading to declines in dopamine and homovanillic acid concentrations in the striatum $[20,22]$. Thalamostriate fibers originate from the intralaminar and centromedian portions of the thalamus that have the greatest neuronal loss [1] and may also contribute to the declines in ICMRGlc seen in the striatum. Afferents to the thalamus are also affected. Fibers projecting from the dentate nucleus through the superior cerebellar peduncle to the ventral lateral thalamus are damaged, as evidenced by the prominent demyelination of this tract $[1,15]$. Thus, declines of glucose metabolism in the caudate nucleus, putamen, and thalamus reflect both loss of neurons and deafferentation.

In the cerebellar cortex in patients with PSP there is a slight to moderate loss of Purkinje cells, and torpedoes have occasionally been noted [1]. Most neuronal loss, however, appears to be limited to the dentate nucleus $[3,15]$, which receives fibers from but does not send fibers to the cerebellar cortex. As expected, we found little change in ICMRGlc in the cerebellar hemispheres and no change in the cerebellar vermis. The asymmetry of cerebellar hemisphere metabolism in normal subjects and the loss of this asymmetry in patients with PSP is of uncertain significance. Previous studies at our center, which included normal subjects of younger age, also demonstrated asymmetry of cerebellar hemisphere glucose metabolic rates [14].

The cerebral cortex generally appears to be spared in PSP when examined by standard neuropathological techniques $[1,6]$. Neurofibrillary tangles have been seen in the cerebral cortex of occasional patients, but this has not been accompanied by any apparent loss of cortical neurons [23]. Because careful morphometric studies of the cerebral cortex have not yet been per-

Table 2. Difference in Glucose Metabolic Rates $(\mathrm{mg} / 100 \mathrm{gm} / \mathrm{min})$ of Left and Right Brain Structures in Progressive Supranuclear Palsy and Normal Control Subjects ${ }^{a}$

\begin{tabular}{lcccc} 
& \multicolumn{2}{c}{ Normal Control Subjects $(\mathrm{n}=21)$} & PSP $(\mathrm{n}=\mathrm{I4})$ \\
\cline { 2 - 5 } Brain Region & Left-Right & $p$ Value & Left-Right & $p$ Value \\
\hline Cerebral cortex & $-0.02 \pm 0.13$ & 0.53 & $-0.20 \pm 0.20$ & 0.002 \\
Cerebellar cortex & $0.18 \pm 0.17$ & 0.0001 & $0.10 \pm 0.42$ & 0.40 \\
Caudate & $0.08 \pm 0.62$ & 0.58 & $0.23 \pm 0.74$ & 0.28 \\
Putamen & $-0.04 \pm 0.45$ & 0.66 & $-0.06 \pm 0.35$ & 0.56 \\
Thalamus & $-0.12 \pm 0.30$ & 0.08 & $-0.17 \pm 0.37$ & 0.10 \\
\hline
\end{tabular}

${ }^{2}$ Values represent means \pm standard deviations; $p$ values represent comparisons by Student's paired $t$ test.

$\mathrm{PSP}=$ progressive supranuclear palsy. 
formed, however, loss of some neurons cannot be excluded. Many afferent projections to the cerebral cortex are damaged, and this may account for the changes in ICMRGlc observed in this study. The nucleus basalis of Meynert, which suffers significant neuronal loss in PSP [24], projects diffusely to the cerebral cortex. A decline in cerebral cortical choline acetyltransferase in PSP is attributable to degeneration of projections from this structure [20]. The locus ceruleus, as mentioned above, shows significant neuronal loss and also projects diffusely to the cerebral cortex. The asymmerry of hypometabolism in the cortex suggests that subcortical structures may also be asymmetrically involved, at least in earlier stages of the illness. Although a decrease in 1CMRGlc in the cerebral cortex generally may result from deafferentation, the prominence of frontal lobe hypometabolism is more difficult to explain. The thalamus, nucleus basalis of Meynert, globus pallidus, and other subcortical structures project somatotopically to the cortex, and conceivably, localized damage to one or more of these structures could account for the pattern of frontal hypometabolism that we observed. Until more is known about the distribution of neuronal loss in subcortical structures that project to the frontal lobe, it is difficult to know whether deafferentation is sufficient to explain the pattern of hypometabolism observed in the cerebral cortex.

Some symptoms of PSP can be predicted on the basis of neuropathological observations. Prominent extrapyramidal signs that in some cases lead to a misdiagnosis of Parkinson's disease [5] result from functional impairment of the basal ganglia. Dysphagia, dysarthria, and some of the disturbance of ocular motility are likely the result of damage to brainstem nuclei and tracts. Ataxia is due to damage of cerebellar efferent pathways. Other symptoms, however, cannot be adequately explained by postmortem studies, and PET provides a better understanding of their pathogenesis.

Patients with PSP exhibit a variety of behavioral and intellectual changes that classically have been localized to the cerebral cortex. Apathy or depression, memory loss, dysphasia, impaired judgment, and perseveration all may be observed in patients with this disease $[7,8$, $25-27]$. The concept of subcortical dementia was developed to account for these changes in the absence of clear neuropathological change in the cerebral cortex [7]; however, recently the distinction between subcortical and cortical dementias has become blurred [28]. FDG-PET now provides evidence that in PSP there is functional impairment in the cerebral cortex and that this may account for some of the intellectual and behavioral changes observed in these patients. Glucose hypometabolism is most prominent in the frontal lobe, and many symptoms attributable to frontal lobe dysfunction are seen in patients with $\operatorname{PSP}[8,9,27]$. Al- though in relative terms the frontal cortex is must affected, glucose metabolic activity is decreased throughout the cerebral cortex. Likewise, impairments of behavior and mentation seen in PSP are not limited to those expected with frontal lobe dysfunction but include a range of cognitive abilities such as dysphasia [29], word-finding difficulty [26], and visuospatial performance $[25-27]$ that have been localized to the asso ciation cortex of both hemispheres. Regions of the cerebral cortex that are most impaired in other dementias, such as Alzheimer's disease, are also affected in PSP. Thus, the constellation of symptoms seen in PSP overlaps with those observed in other kinds of demen. tia $[8,30]$. The types of symptoms occurring in PSP are related to the severity of illness and vary somewhat between individuals. This accounts for the difficulty encountered in identifying a characteristic psychometric profile for patients with PSP.

In the present study, the glucose metabolic rate was most impaired in the cerebral cortex just anterior to the central sulcus. Although it is not possible to localize this region with certainty, it likely encompasses supplementary motor areas and the frontal eye fields in the frontal cortex. Functional imparment of these regions is likely to result in difficulty with initiation and performance of motor tasks such as those observed in patients with PSP [31]. Impairment of the frontal eye fields may contribute to the abnormalities in ocular motility that are so characteristic of this disorder. Animal studies indicate that voluntary control of saccadic eye movements depends upon both the superior colliculus and the frontal eye fields. Ablation of either alone in trained monkeys in the chronic state produces only subtle changes in saccadic eye movements [32]. Combined lesions of the superior colliculus and frontal eye fields, however, cause devastating oculomotor abnormalities. Such animals have saccadic paralysis and appear to lose the ability to move their eyes voluntarily, although they can do so reflexively [33]. 1-Methyl4-phenyl-1,2,3,6-tetrahydropyridine (MPTP)-induced parkinsonism in monkeys has been found to decrease glucose metabolism in the paralamellar mediodorsal thalamus and in the frontal eye tields. These declines are reversed by the administration of levodopa, which suggests that they may be dopaminergic in origin $\{34\}$. In these monkeys, oculomotor abnormalities were also reversed by levodopa. Levodopa therapy does not usually improve eye movements in patients with PSP, and thus it is not clear whether a similar mechanism can account for our findings.

Declines in the metabolism of the frontal cortex are the most apparent changes observed when FDG-PET is used in patients with PSP. This pattern is quite distinctive from that seen in Alzheimer's discase where more posterior regions of the cerebral cortex are most impaired, and the frontal cortex is relatively spared 
[10]. Hypometabolism, most prominent in the frontal cortex, has also been observed in Parkinson's disease uncomplicated by dementia [35] and Pick's disease [36, 37]; however, no declines of glucose metabolism in brainstem, basal ganglia, or thalamus were observed in these disorders. FDG-PET is helpful in differentiating PSP from other diseases leading to gait instability such as olivopontocerebellar atrophy (OPCA). Distinguishing these two diseases can be difficult, particularly in the early stages of the illness and in view of the observation of supranuclear gaze palsy in some patients with OPCA [38]. Patients with OPCA show hypometabolism in the cerebellar hemispheres and vermis and in the brainstem but have normal values in the thalamus and cerebral cortex [14, 39], a pattern quite distinct from that observed in patients with PSP.

In the present study, rate constants derived from normal subjects have been used in computing ICMRGlc. The applicability of these rate constants in disease states such as PSP has not yet been tested. We have made no corrections for atrophy, although atrophy of the pontine tegmentum is often apparent at gross examination of the brain after death $[3,15]$ and has been noted using high-resolution CT [40-42]. Atrophy of the cerebellum or the cerebral cortex has been demonstrated with CT $[41,42]$ and pneumoencephalography [43], although atrophy of these structures is not clearly recognizable at gross postmortem examination. If atrophy causes loss of volume in brain structures being measured with PET and if this volume change is significant relative to the resolution of the scanner, a decline in the recovery coefficient could also lead to an underestimation of glucose metabolism in these structures $[44,45]$. Atrophy may also complicate the measurement of subcortical structures. We have chosen a method that centers a uniform region of interest over the peak activity corresponding to a subcorrical structure, rather than attempting to outline the entire extent of the structure based on its anatomy or appearance on the image. This should minimize the error due to difficulties in visualizing a hypometabolic structure. The significance of atrophy in our studies, however, is not known. Even if problems with rate constants and partial volume effects are found to make a difference in the interpretation of the present results, these factors do not negate the diagnostic utility of our observations.

Patients with PSP demonstrate a distinctive pattern of glucose hypometabolism that accounts for many of the characteristic features of this disease. The extent to which this pattern results from intrinsic neuronal damage or deafferentation cannot at present be determined; however, FDG-PET contributes to our understanding of this disease and in the future may play an important role in improving the accuracy of clinical diagnosis.
Supported in part by the Louise Madsen Fund, the Campbell Foundation, and Grant NS-15655 from the National Institute of Neurological and Communicative Disorders and Stroke. Dr Foster is a recipient of a Clinical Investigator Development Award (NS01023) from the National Institutes of Health

Presented in part at the Seventh Annual Meeting of the American Society for Neurological Investigation, San Francisco, California, Oct 18, 1987, and at the 112th Annual Meeting of the American Neurological Association, San Francisco, CA, Oct 18-21, 1987.

\section{References}

1. Steele JC, Richardson JC, Olszewski J. Progressive supranuclear palsy. Arch Neurol 1964;10:333-359

2. Kristensen MO. Progressive supranuclear palsy -20 years later. Acta Neurol Scand 1985;71:177-189

3. Steele JC. Progressive supranuclear palsy. Brain 1972;95:693704

4. Maher ER, Lees AJ. The clinical features and natural history of the Steele-Richardson-Olszewski syndrome (progressive supranuclear palsy). Neurology 1986;36:1005-1008

5. Jankovic J. Progressive supranuclear palsy: clinical and pharmacologic update. Neurol Clin 1984:2:473-486

6. Jellinger K. Progressive supranuclear palsy (subcortical argyrophilic dystrophy). Acta Neuropathol 1971;19:347-352

7. Albert ML, Feldman RG, Willis AL. The "subcortical dementia" of progressive supranuclear palsy. J Neurol Neurosurg Psychiatry $1974 ; 37: 121-130$

8. Pillon B, Dubois B, Lhermitte F, Agid Y. Heterogeneity of cognitive impairment in progressive supranuclear palsy, Parkinson's disease, and Alzheimer's disease. Neurology 1986;36: 1179-1185

9. D'Antona R, Baron JC, Samson Y, et al. Subcortical dementia: frontal cortex hypometabolism detected by positron tomography in patients with progressive supranuclear palsy. Brain 1985;108:785-799

10. Foster NL, Gilman S, Berent S, Hichwa RD. Distinctive patterns of cerebral cortical glucose metabolism in progressive supranuclear palsy and Alzheimer's disease studied with positron emission tomography. Neurology 1986;36 (4 suppl 1):338

11. Foster NL, Gilman S, Berent S, et al. Glucose hypometabolism in progressive supranuclear palsy is not limited to frontal cortex. Ann Neurol 1987;22:123

12. Bida G, Satyamurthy N, Barrio J. The synthesis of 2-[F-18] fluoro-2-deoxy-D-glucose using glycols: a reexamination. $\mathrm{J} \mathrm{Nu}$ clear Med 1984;25:1327-1334

13. Hawkins RA, Mazziotta JC, Phelps ME, et al. Cerebral glucose netabolism as a function of age in man: influence of the rate constants in the fluorodeoxyglucose method. J Cereb Blood Flow Metab 1983;3:250-253

14. Gilman S, Markel DS, Koeppe RA, et al. Cerebellar and brainstem hypometabolism in olivopontocerebellar atrophy detected with positron emission tomography. Ann Neurol 1988;23:223230

15. Behrman S, Carroll JD, Janota I, Matthews WB. Progressive supranuclear palsy. Clinico-pathological study of four cases. Brain 1969;92:663-678

16. Sokoloff L. Cerebral circulation, energy metabolism, and protein synthesis: General characteristics and principles of measurement. In: Phelps ME, Mazziotta JC, Schelbert HR, eds. Positron emission tomography: principles and applications for the brain and heart. New York: Raven, 1986:1-72

17. Phelps ME, Mazziotta JC, Huang SC. Study of cerebral function with positron computed tomography. J Cereb Blood Flow Metab 1982;2:113-162 
18. Blumenthal $\mathrm{H}$, Miller $\mathrm{C}$. Motor nuclear involvement in progressive supranuclear palsy. Arch Neurol 1969;20:362-367

19. Ishino $\mathrm{H}$, Higashi $\mathrm{H}$, Kuroda $\mathrm{S}$, et al. Motor nuclear involvement in progressive supranuclear palsy. J Neurol Sci 1974; 22:235-244

20. Ruberg M, Javoy-Agid F, Hirsch E, et al. Dopaminergic and cholinergic lesions in progressive supranuclear palsy. Ann Neurol 1985;18:523-529

21. Baron JC, Maziere B, Loch C, et al. Loss of striatal $\left({ }^{76} \mathrm{Br}\right)$ bromospiperone binding sites demonstrated by positron tomography in progressive supranuclear palsy. J Cereb Blood Flow Metab 1986;6:131-136

22. Kish SJ, Chang LJ, Mirchandani L, et al. Progressive supranuclear palsy: relationship between extrapyramidal disturbances, dementia, and brain neurotransmitter markers. Ann Neurol 1985;18:530-536

23. Ishino H, Otsuki S. Frequency of Alzheimer's neurofibrillary tangles in the cerebral cortex in progressive supranuclear palsy (subcortical argyrophilic dystrophy). J Neurol Sci 1976;28:309316

24. Tagliavini F, Pilleri G, Bouras C, Constantinidis J. The basal nucleus of Meynert in patients with progressive supranuclear palsy. Neurosci Lett 1984;44:37-42

25. Fisk JD, Goodale MA, Burkhart G, Barnett HJM: Progressive supranuclear palsy: the relationship between ocular motor dysfunction and psychological test performance. Neurology 1982; 32:698-705

26. Maher ER, Smith EM, Lees AJ. Cognitive deficits in the SteeleRichardson-Olszewski syndrome (progressive supranuclear palsy). J Neurol Neurosurg Psychiatry 1985;48:1234-1239

27. Kimura D, Barnett HJM, Burkhart G. The psychological test pattern in progressive supranuclear palsy. Neuropsychologia 1981;19:301-306

28. Whitehouse PJ. The concept of subcortical and cortical dementia: another look. Ann Neurol 1986;19:1-6

29. Perkin GD, Lees AJ, Stern GM, Kocen RS. Problems in the diagnosis of progressive supranuclear palsy (Steele-RichardsonOlszewski syndrome). Can J Neurol Sci 1978;5:167-173

30. Berent S, Foster NL, Gilman S, et al. Patterns of cortical ${ }^{18} \mathrm{~F}$ FDG metabolism in Alzheimer's and progressive supranuclear palsy patients are related to the types of cognitive impairments. Neurology 1987;37 (suppl 1):172
31. Rafal RD, Grimm RJ. Progressive supranuclear palsy: functional analysis of the response to methysergide and antiparkinsonian agents. Neurology 1981;31:1507-1518

32. Wurtz RH, Goldberg ME. Activity of superior colliculus in behaving monkey. IV. Effects of lesions on eye movements. J Neurophysiol 1972;35:587-596

33. Schiller PH, True SD, Conway JL. Deficits in eye movements following frontal eye field and superior colliculus ablations. J Neurophysiol 1980;44:1175-1189

34. Ho VW, Porrino LJ, Crane AM, et al. Merabolic mapping of oculomotor system in MPTP-induced parkinsonian monkeys. Ann Neurol 1988;23:86-89

35. Perlmutter JS, Raichle ME. Regional blood tow in hemiparkinsonism. Neurology 1985;35:1127-1134

36. Friedland RP, Jagust WJ, Ober BA, et al. The pathophysiology of Pick's disease: a comprehensive case study. Neurology 1986; 36:268-269

37. Kamo H, McGeer PL, Harrop R, et al. Positron emission tomography and histopathology in Pick's disease. Neurology 1987;37:439-445

38. Koeppen AH, Hans MB. Supranuclear ophthalmoplegia in olivopontocerebellar degeneration. Neurology 1976;26:764-768

39. Gilman S, Markel DS, Koeppe R, et al. Cerebellar hypometabolism in olivopontocerebellar atrophy detected by positron emission tomography. Neurology 1986;36:230

40. Abe S, Miyasaka K, Tashiro K, et al. Evaluation of the brainstem with high-resolution $\mathrm{CT}$ in cerebellar atrophic processes. AJNR 1983;4:446-449

41. Ambrosetto P, Michelucci R, Forti A, Tassinari CA. CT findings in progressive supranuclear palsy. J Comput Assist Tomogr 1984;8:406-409

42. Haldeman S, Goldman JW, Hyde J, Pribram HFW. Progressive supranuclear palsy, computed tomography, and response to antiparkinsonian drugs. Neurology 1981;31:442-445

43. Bentson JR, Keesey JC. Pneumoencephalography of progressive supranuclear palsy. Radiology 1974;113:89-94

44. Mazziotta JC, Phelps ME, Plummer D, Kuhl DE. Quantitation in positron emission computed tomography: 5. Physical-anatomical effects. J Comput Assist Tomogr 1981;5:734-743

45. Herscovitch P, Auchus AP, Gado M, et al. Correction of positron emission tomography data for cerebral atrophy. J Cereb Blood Flow Metab 1986;6:120-124 